Article

\title{
Antibacterial Activity of Long-Chain Polyunsaturated Fatty Acids against Propionibacterium acnes and Staphylococcus aureus
}

\author{
Andrew P. Desbois * and Keelan C. Lawlor \\ Marine Biotechnology Research Group, Institute of Aquaculture, School of Natural Sciences, \\ University of Stirling, Stirlingshire, Scotland FK9 4LA, UK; E-Mail: keelan107@gmail.com \\ * Author to whom correspondence should be addressed; E-Mail: ad54@stir.ac.uk; \\ Tel.: +44-1786-467-894; Fax: +44-1786-472-133.
}

Received: 24 September 2013; in revised form: 31 October 2013 / Accepted: 4 November 2013 / Published: 13 November 2013

\begin{abstract}
New compounds are needed to treat acne and superficial infections caused by Propionibacterium acnes and Staphylococcus aureus due to the reduced effectiveness of agents used at present. Long-chain polyunsaturated fatty acids (LC-PUFAs) are attracting attention as potential new topical treatments for Gram-positive infections due to their antimicrobial potency and anti-inflammatory properties. This present study aimed to investigate the antimicrobial effects of six LC-PUFAs against $P$. acnes and S. aureus to evaluate their potential to treat infections caused by these pathogens. Minimum inhibitory concentrations were determined against $P$. acnes and $S$. aureus, and the LC-PUFAs were found to inhibit bacterial growth at $32-1024 \mathrm{mg} / \mathrm{L}$. Generally, $P$. acnes was more susceptible to the growth inhibitory actions of LC-PUFAs, but these compounds were bactericidal only for $S$. aureus. This is the first report of antibacterial activity attributed to 15-hydroxyeicosapentaenoic acid (15-OHEPA) and 15-hydroxyeicosatrienoic acid (HETrE), while the anti-P. acnes effects of the six LC-PUFAs used herein are novel observations. During exposure to the LC-PUFAs, S. aureus cells were killed within 15-30 min. Checkerboard assays demonstrated that the LC-PUFAs did not antagonise the antimicrobial potency of clinical agents used presently against $P$. acnes and $S$. aureus. However, importantly, synergistic interactions against $S$. aureus were detected for combinations of benzoyl peroxide with 15-OHEPA, dihomo- $\gamma$-linolenic acid (DGLA) and HETrE; and neomycin with 15-OHEPA, DGLA, eicosapentaenoic acid, $\gamma$-linolenic acid and HETrE. In conclusion, LC-PUFAs warrant further evaluation as possible new agents to
\end{abstract}


treat skin infections caused by $P$. acnes and $S$. aureus, especially in synergistic combinations with antimicrobial agents already used clinically.

Keywords: acne vulgaris; benzoyl peroxide; C20:5n-3; combination therapy; docosahexaenoic acid (DHA); natural products; omega-3; skin infection; synergy

\section{Introduction}

Colonisation of the skin by the anaerobic Gram-positive bacterium Propionibacterium acnes is one factor involved in the aetiology of acne vulgaris [1,2]. In addition, colonisation by Staphylococcus aureus, an opportunistic Gram-positive pathogen, has been implicated in the pathogenesis of acne [1], and this bacterium can also cause superficial skin infections such as boils, pimples and impetigo [3]. Topical treatment and management of acne relies on the application of benzoyl peroxide (BPO), salicylic acid (SA) and certain antibiotics, while topical $S$. aureus infections are typically treated with fusidic acid (FUS), mupirocin (MUP), neomycin (NEO) and polymyxin B (POL) [4]. However, there has been a decrease in the clinical efficacy of many treatment agents during the last 20 years, perhaps due to the increasing prevalence of drug-resistant $P$. acnes and $S$. aureus strains [5-8]. Moreover, some of these agents are associated with undesirable side effects such as skin irritation [4], meaning that there is a clinical need to identify alternative compounds with improved therapeutic properties for topical infections caused by $P$. acnes and $S$. aureus.

Fatty acids are attracting attention as potential therapeutic antimicrobial agents due to their potency, broad spectrum of activity and the lack of classical resistance mechanisms against the actions of these compounds [9,10]. In particular, various long-chain polyunsaturated fatty acids (LC-PUFAs), which are found naturally at high levels in many marine organisms [11], have been shown to exert highly potent activity against Gram-positive bacteria, including eicosapentaenoic acid (EPA; C20:5n-3) [12,13], docosahexaenoic acid (DHA; C22:6n-3) [14-17], $\gamma$-linolenic acid (GLA; C18:3n-6) [15,18-21] and dihomo- $\gamma$-linolenic acid (DGLA; C20:3n-6) [15]. Furthermore, LC-PUFAs deserve special consideration for topical application because they are often associated with anti-inflammatory properties, which may provide supplementary benefit during therapy $[10,22,23]$. Thus, the aim of this present study was to investigate the antimicrobial effects of six LC-PUFAs (DGLA, DHA, EPA, GLA, 15-hydroxyeicosatrienoic acid [HETrE], and 15-hydroxyeicosapentaenoic acid [15-OHEPA]) against $P$. acnes and $S$. aureus, to evaluate the potential of these compounds for treating topical infections caused by these pathogens.

\section{Results}

\subsection{Antibacterial Activity of Six LC-PUFAs against P. acnes and S. aureus}

Minimum inhibitory (MIC) and bactericidal (MBC) concentrations for six LC-PUFAs (DHA, EPA, GLA, DGLA, HETrE and 15-OHEPA) were determined by broth micro-dilution against P. acnes NCTC737 and $S$. aureus ATCC43300. In all tests, ethanol and dimethyl sulfoxide (DMSO) carrier solvents had no detectable effect on the growth of these test bacteria. HETrE and DHA were the most 
effective LC-PUFAs for inhibiting the growth of $P$. acnes (MIC $=32 \mathrm{mg} / \mathrm{L}$ ) followed by GLA $(\mathrm{MIC}=64 \mathrm{mg} / \mathrm{L})($ Table 1$)$. None of the LC-PUFAs killed P. acnes up to the maximum concentration tested $(4096 \mathrm{mg} / \mathrm{L})$. Meanwhile, DHA and EPA were the most effective LC-PUFAs against $S$. aureus $(\mathrm{MICs}=128 \mathrm{mg} / \mathrm{L})$, but the LC-PUFAs were generally less effective against this bacterium. Indeed, the MIC for each LC-PUFA was up to eight-fold greater against $S$. aureus compared with $P$. acnes (Table 1); however, in contrast to observations for $P$. acnes, each of the six LC-PUFAs was bactericidal against $S$. aureus and MBC values were equal to MIC or $2 \times$ MIC (Table 1). Next, to assess interstrain variability in susceptibility to the LC-PUFAs, $\mathrm{MIC}_{50}$ and $\mathrm{MBC}_{50}$ values were determined using ten isolates of $S$. aureus from diverse backgrounds, including clinical and laboratory strains and isolates with resistance to methicillin and vancomycin (Table 2). $\mathrm{MIC}_{50}$ and $\mathrm{MBC}_{50}$ values correspond to the concentrations required to inhibit or kill $50 \%$ of the strains tested, respectively. The relative potency of the LC-PUFAs against these $S$. aureus isolates was: DHA $>$ EPA $>$ GLA $>$ HETrE $>$ 15-OHEPA $=$ DGLA (Table 2).

Table 1. MICs and MBCs against P. acnes NCTC737 and S. aureus ATCC43300 for six LC-PUFAs.

\begin{tabular}{cccccc}
\hline \multirow{2}{*}{ Compound } & \multicolumn{2}{c}{ P. acnes } & & \multicolumn{2}{c}{ S. aureus } \\
\cline { 2 - 3 } \cline { 5 - 6 } & MIC (mg/L) & MBC (mg/L) & & MIC (mg/L) & MBC (mg/L) \\
\hline DGLA & 128 & $>4096$ & & 1024 & 1024 \\
DHA & 32 & $>4096$ & & 128 & 128 \\
EPA & 128 & $>4096$ & & 128 & 256 \\
GLA & 64 & $>4096$ & & 512 & 512 \\
HETrE & 32 & $>4096$ & & 256 & 512 \\
15-OHEPA & 128 & $>4096$ & & 512 & 1024 \\
\hline
\end{tabular}

Table 2. $\mathrm{MIC}_{50}$ and $\mathrm{MBC}_{50}$ values for six LC-PUFAs against ten diverse isolates of S. aureus.

\begin{tabular}{ccc}
\hline \multirow{2}{*}{ Compound } & \multicolumn{2}{c}{ S. aureus } \\
\cline { 2 - 3 } & MIC $_{\mathbf{5 0}} \mathbf{( m g / \mathbf { L } )}$ & $\mathbf{M B C}_{\mathbf{5 0}} \mathbf{( \mathbf { m g } / \mathbf { L } )}$ \\
\hline DGLA & 1024 & 2048 \\
DHA & 128 & 256 \\
EPA & 256 & 256 \\
GLA & 512 & 512 \\
HETrE & 512 & 1024 \\
15-OHEPA & 1024 & 2048 \\
\hline
\end{tabular}

\subsection{Killing Kinetics of Six LC-PUFAs against $\mathrm{S}$. aureus}

The killing kinetics of the six LC-PUFAs were determined at $1 \times \mathrm{MBC}$ against $\sim 2.6 \times 10^{5}$ colony forming unit (CFU)/mL inoculums of $S$. aureus ATCC43300 prepared in phosphate-buffered saline (PBS). The incubations were performed at $37^{\circ} \mathrm{C}$ and there was little change in the viability of cells in the control group that were exposed only to the carrier solvents, ethanol and DMSO. Importantly, the LC-PUFAs killed the $S$. aureus inoculums rapidly and no viable bacteria were detected within $15 \mathrm{~min}$ 
of initial exposure for five of the six LC-PUFAs, with DGLA taking just 30 min to completely kill the inoculum (Figure 1).

Figure 1. Killing kinetics of six LC-PUFAs against a $S$. aureus ATCC43300 inoculum of $\sim 2.6 \times 10^{5}$ colony forming unit $(\mathrm{CFU}) / \mathrm{mL}$ in phosphate-buffered saline (PBS) showing that each LC-PUFA killed the bacteria rapidly. $n=3$; mean \pm one standard deviation (not all error bars are visible). Please note that a single kill curve is shown for DHA, EPA, HETrE and 15-OHEPA because the values were identical and the kill curves overlapped exactly.

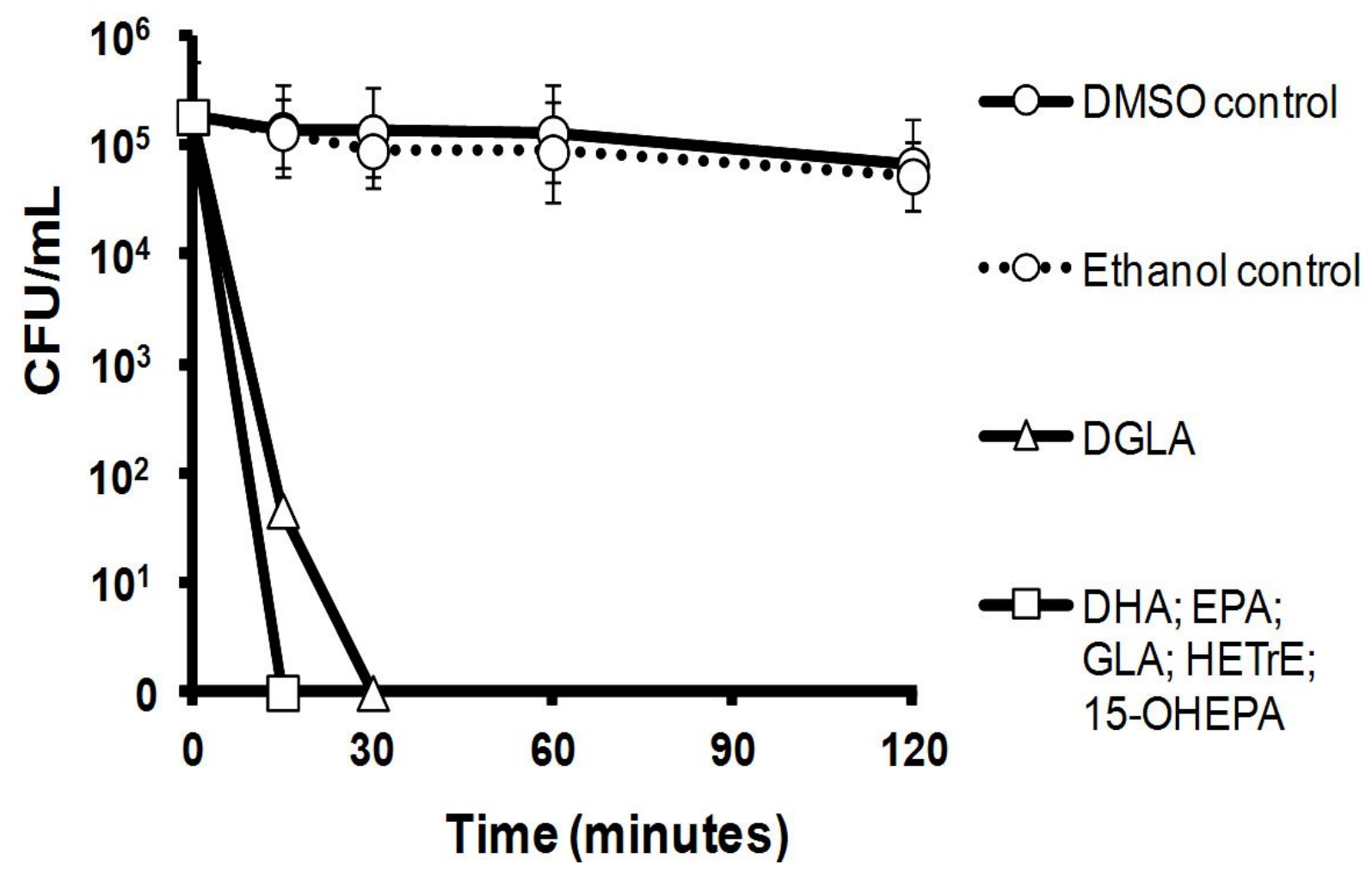

\subsection{Efficacy of Six LC-PUFAs in Combination with Clinical Antimicrobial Agents}

Combination therapies are often prescribed during the treatment of acne vulgaris and topical staphylococcal infections $[1,2,4]$ because this approach can improve clinical outcome and may also reduce the opportunity to select for drug-resistant strains [24]. Therefore, to assess whether the LC-PUFAs could be used without reducing the efficacy of agents currently prescribed to treat acne and topical staphylococcal infections, checkerboard tests were performed for each LC-PUFA in combination with six topical antimicrobials. Moreover, these tests enabled us to evaluate whether any of the combinations acted in synergy, as synergistic combinations of antimicrobial agents can deliver enhanced efficacy through greater potency and also reduce undesirable side effects due to the application of lower doses of individual agents [24]. Initially, MICs and MBCs had to be determined against $P$. acnes and $S$. aureus for two antimicrobial agents used to treat acne vulgaris (BPO and SA) and additionally against $S$. aureus for four antibiotics used clinically in the treatment of topical staphylococcal infections (FUS, MUP, NEO and POL) (Table 3). The MIC against P. acnes for BPO and SA was $64 \mathrm{mg} / \mathrm{L}$ but these agents failed to kill the bacterium up to $4096 \mathrm{mg} / \mathrm{L}$, which was the 
maximum concentration tested (Table 3). Against $S$. aureus, the MIC and MBC for BPO was $512 \mathrm{mg} / \mathrm{L}$, while the MIC and MBC for SA was $512 \mathrm{mg} / \mathrm{L}$ and $1024 \mathrm{mg} / \mathrm{L}$, respectively, meaning that these agents were less potent against $S$. aureus compared with $P$. acnes. FUS and MUP were the most potent clinical agents against $S$. aureus $(\mathrm{MICs}=0.25 \mathrm{mg} / \mathrm{L})$, while POL and NEO were less effective (Table 3). Nevertheless, all six clinical antimicrobials exhibited bactericidal activity against $S$. aureus at minimum concentrations up to $64 \times$ MIC (Table 3 ).

Table 3. MICs and MBCs against P. acnes NCTC737 and S. aureus ATCC43300 for various clinical antimicrobial agents used to treat acne or superficial topical staphylococcal infections. $\mathrm{n} / \mathrm{d}$, not determined.

\begin{tabular}{cccccc}
\hline \multirow{2}{*}{ Compound } & \multicolumn{2}{c}{ P. acnes } & & \multicolumn{2}{c}{ S. aureus } \\
\cline { 2 - 3 } \cline { 5 - 6 } & MIC (mg/L) & MBC $(\mathbf{m g} / \mathbf{L})$ & & MIC (mg/L) & MBC (mg/L) \\
\hline BPO & 64 & $>4096$ & & 512 & 512 \\
FUS & $\mathrm{n} / \mathrm{d}$ & $\mathrm{n} / \mathrm{d}$ & & 0.25 & 8 \\
MUP & $\mathrm{n} / \mathrm{d}$ & $\mathrm{n} / \mathrm{d}$ & & 0.25 & 16 \\
NEO & $\mathrm{n} / \mathrm{d}$ & $\mathrm{n} / \mathrm{d}$ & & 32 & 128 \\
POL & $\mathrm{n} / \mathrm{d}$ & $\mathrm{n} / \mathrm{d}$ & & 16 & 128 \\
SA & 64 & $>4096$ & & 1024 & 2048 \\
\hline
\end{tabular}

Using the pre-determined MIC values, checkerboard assays were performed to detect the interactions between the LC-PUFAs and the clinical antimicrobial drugs against the growth of $P$. acnes and $S$. aureus. The fractional inhibitory concentration (FIC) on duplicate checkerboards was used to calculate a mean FIC for each interaction, and mean FIC $\leq 0.5$ was defined as synergy while mean FIC $\geq 4$ was defined as antagonism [25]. None of the combinations tested against $P$. acnes gave mean FICs of $\leq 0.5$ or $\geq 4$, indicating no growth inhibitory synergy or antagonism, respectively (Table 4). Against $S$. aureus, none of the combinations gave a mean FIC $\geq 4$, indicating no antagonism of efficacy between the LC-PUFAs and the clinical antimicrobial agents (Table 5). Interestingly, several combinations of LC-PUFAs and clinical agents showed antimicrobial synergy against $S$. aureus, including BPO with 15-OHEPA, DGLA and HETrE; and, most notably, NEO with five of the six LC-PUFAs, with the exception being DHA that had an FIC of 0.56 meaning that it only just failed to meet the definition of synergy (Table 5).

Table 4. Mean FIC values of interactions between six LC-PUFAs and two topical antimicrobial agents against $P$. acnes NCTC737 showing that none of the combinations were synergistic or antagonistic. $n=2$; mean \pm one standard deviation.

\begin{tabular}{ccccccc}
\hline $\boldsymbol{P}$.acnes & DGLA & DHA & EPA & GLA & HETrE & 15-OHEPA \\
\hline BPO & $1.50 \pm 0.71$ & $1.50 \pm 0.71$ & $1.50 \pm 0.71$ & $1.50 \pm 0.71$ & $0.75 \pm 0.00$ & $0.88 \pm 0.18$ \\
SA & $2.00 \pm 0.00$ & $1.13 \pm 0.53$ & $0.78 \pm 0.31$ & $1.50 \pm 0.71$ & $1.75 \pm 0.35$ & $2.00 \pm 0.00$ \\
\hline
\end{tabular}


Table 5. Mean FIC values of interactions between six LC-PUFAs and six topical antimicrobial agents against $S$. aureus ATCC43300 showing that there was synergy $($ FIC $\leq 0.5)$ between BPO with three LC-PUFAs and NEO with five LC-PUFAs. Values in bold indicate synergy; $n=2$; mean \pm one standard deviation.

\begin{tabular}{ccccccc}
\hline S. aureus & DGLA & DHA & EPA & GLA & HETrE & 15-OHEPA \\
\hline BPO & $\mathbf{0 . 4 4} \pm \mathbf{0 . 0 9}$ & $2.00 \pm 0.00$ & $1.00 \pm 0.00$ & $0.56 \pm 0.09$ & $\mathbf{0 . 5 0} \pm \mathbf{0 . 0 0}$ & $\mathbf{0 . 4 4} \pm \mathbf{0 . 0 9}$ \\
SA & $2.00 \pm 0.00$ & $1.25 \pm 0.35$ & $0.88 \pm 0.18$ & $0.88 \pm 0.18$ & $0.88 \pm 0.18$ & $1.00 \pm 0.00$ \\
FUS & $0.81 \pm 0.27$ & $2.00 \pm 0.00$ & $1.13 \pm 0.18$ & $1.50 \pm 0.71$ & $1.50 \pm 0.71$ & $2.00 \pm 0.00$ \\
MUP & $1.50 \pm 0.71$ & $1.50 \pm 0.00$ & $2.00 \pm 0.00$ & $2.25 \pm 0.35$ & $2.00 \pm 0.00$ & $1.38 \pm 0.88$ \\
NEO & $\mathbf{0 . 2 8} \pm \mathbf{0 . 1 3}$ & $0.56 \pm 0.09$ & $\mathbf{0 . 3 8} \pm \mathbf{0 . 0 0}$ & $\mathbf{0 . 1 9} \pm \mathbf{0 . 0 0}$ & $\mathbf{0 . 3 8} \pm \mathbf{0 . 0 0}$ & $\mathbf{0 . 3 4} \pm \mathbf{0 . 0 4}$ \\
POL & $1.78 \pm 1.72$ & $2.00 \pm 0.00$ & $2.00 \pm 0.00$ & $1.06 \pm 0.62$ & $0.75 \pm 0.00$ & $0.94 \pm 0.27$ \\
\hline
\end{tabular}

\section{Discussion}

New treatments are required for topical infections caused by $P$. acnes and $S$. aureus as the efficacy of many treatments has reduced due to drug resistance and undesirable side effects can also cause problems for patients. In this present study, the antimicrobial effects of six LC-PUFAs were investigated and each compound potently inhibited the growth of $P$. acnes and $S$. aureus in vitro. Moreover, each LC-PUFA was bactericidal against $S$. aureus and caused cell death within just 30 min. Notably, none of the LC-PUFAs antagonised the antimicrobial activity of agents used to treat topical infections caused by these two pathogens; however, various combinations of LC-PUFAs with BPO or NEO acted synergistically to inhibit the growth of $S$. aureus.

Though LC-PUFAs are known to be potently antibacterial [9], to our knowledge this is the first time that 15-OHEPA and HETrE have been shown to exert antimicrobial activities. Furthermore, this is the first study to report that the six LC-PUFAs tested herein are detrimental for the growth of $P$. acnes, though other fatty acids are known to be toxic to this bacterium, including $\alpha$-linolenic acid (C18:3n-3) [26] and lauric acid (C12:0) [27]. Despite LC-PUFAs lacking bactericidal action against P. acnes, these compounds did exert similar antibacterial potency as BPO and SA indicating that potency should not prevent LC-PUFAs being considered as alternative agents in acne therapy. The antimicrobial potency of BPO against $P$. acnes in this present study is similar to previous reports, but the lack of bactericidal activity, such as has been reported previously, may stem from methodological differences in quantifying antimicrobial action and the preparation of the inoculum, as well as the use of different growth conditions and bacterial strains [27-29]. Despite no indication of P. acnes resistance to LC-PUFAs, the probability to select for strains with resistance to the action of these compounds warrants investigation, particularly as a large difference was observed between MIC and MBC values that may increase the opportunity for mutant selection [30].

Confirming previous observations, EPA, DHA and GLA were potently antibacterial against S. aureus $[18,21,31]$, but the activity of DGLA against this pathogen is reported here for the first time. Knapp and Melly [32] did show that a C20:3 fatty acid had anti-S. aureus activity, but it was not clear which specific fatty acid isomer was used. The LC-PUFAs were similarly potent against a variety of $S$. aureus strains, suggesting that interstrain susceptibility should not present difficulties during future clinical use. The bactericidal action and small differences between MIC and MBC values against 
$S$. aureus should reduce the chances to select for resistant strains, but this requires experimental confirmation [30].

The LC-PUFAs killed $S$. aureus rapidly with kill times similar to earlier observations for other fatty acids against this bacterium, including caprylic acid (C8:0) [33], lauric acid [34], sapienic acid (C16:1n-10) [35,36], oleic acid (C18:1n-9) [37], GLA [19] and EPA [38]. Such rapid cell death caused by the LC-PUFAs strongly suggests a cell membrane lytic mechanism of action against $S$. aureus, which is in line with previous studies on Gram-positive species (see review by Desbois and Smith [9]). However, the lack of bactericidal action against $P$. acnes even at high concentrations $(4096 \mathrm{mg} / \mathrm{L})$ suggests a different mode of action for LC-PUFAs against this bacterium and growth inhibition could result from disruption at multiple cellular targets [9]. The underlying antimicrobial mechanisms of LC-PUFAs against $P$. acnes merit further investigation.

In checkerboard tests, none of the LC-PUFAs antagonised the efficacy of the topical antimicrobial treatments used against $P$. acnes and $S$. aureus, suggesting that the LC-PUFAs could be added to current treatment regimens without reducing clinical effectiveness. The checkerboards revealed no antimicrobial synergy against $P$. acnes between LC-PUFAs with BPO and SA, even though an earlier study showed that the efficacy of BPO was enhanced in presence of lipid compounds [28]. Against S. aureus, three of the six LC-PUFAs acted in synergy with the anti-acne agent BPO, while five of the LC-PUFAs acted synergistically with the aminoglycoside antibiotic NEO. One major benefit of such antimicrobial combinations is the reduced opportunity to select for resistant bacterial strains [24]. Few studies have examined the interactions of fatty acids and LC-PUFAs with clinical antibiotics against bacteria $[36,39,40]$, but the findings in this present study emphasise the potential importance of testing for such synergism as has been highlighted elsewhere $[9,13]$. At this stage, the nature of the synergy between the LC-PUFAs with BPO or NEO is unknown but one may speculate that they improve the penetration of the clinical agents into the cell because fatty acids can increase cell membrane permeability [41]. The synergy between BPO and certain LC-PUFAs could be exploited to reduce the concentrations of compounds applied to the skin during acne therapy and this could reduce the severity of undesirable side effects. The discovery of synergy between LC-PUFAs and NEO may have uncovered an important new strategy to combat $S$. aureus infections. Synergy has been reported between aminoglycoside antibiotics and a fatty acid-rich oil but few details of the oil composition were given and it was unclear which components were actively involved [42].

Of course, this present study is limited to in vitro observations and in vivo studies are needed to understand the true potential of applying LC-PUFAs in combination with clinical antimicrobial drugs for treating $P$. acnes and $S$. aureus infections. The prospects for using LC-PUFAs to treat $P$. acnes and $S$. aureus infections appear to be promising because other fatty acids have shown topical efficacy in vivo against these pathogens, including lauric acid against $P$. acnes in a murine ear skin infection model [27]; sapienic acid against $S$. aureus in a murine dermatitis model [36]; and oleic acid (C18:1n-9) against topical staphylococcal infections in mice [43,44]. Furthermore, 10-undecylenic acid $(\mathrm{C} 11: 1 n-1)$ is used already as a topical agent for the treatment of fungal infections [45]. Fatty acids are antimicrobial components of the innate immune system found on mammalian skin and the application of exogenous LC-PUFAs during therapy would augment these natural defences [46-50]. Further benefit may be realised because fatty acids can synergise with natural antimicrobial peptides found on human skin [51]. Moreover, reductions in the prescription of conventional antibiotics for topical 
infections, especially systemic agents, will beneficially reduce the opportunity to select for drug-resistant strains in the non-target skin microbiota [1,7].

\section{Experimental Section}

\subsection{Reagents and Bacteria}

All bacterial culture media and reagents were of the highest purity available and purchased from Sigma-Aldrich Ltd. (Poole, UK) unless stated. The molecular structures of the fatty acids used in this present study are shown in Figure 2. Stock solutions of DGLA (97.9\%; Equateq Ltd., Callanish, UK), HETrE (94.3\%; Equateq Ltd., Callanish, UK), 15-OHEPA (50 mg/mL; Sapala Organics Pvt. Ltd., Hyderabad, India) and BPO (75\%) were prepared in DMSO ( $\geq 99.9 \%)$ to $50 \mathrm{mg} / \mathrm{mL}$; DHA, EPA and GLA were prepared in ethanol to $100 \mathrm{mg} / \mathrm{mL}$; POL and FUS were prepared in sterile distilled water to $20 \mathrm{mg} / \mathrm{mL}$; while MUP and SA were prepared in ethanol to $50 \mathrm{mg} / \mathrm{mL}$. NEO was purchased as a 10 $\mathrm{mg} / \mathrm{mL}$ solution in sterile saline. P. acnes NCTC737 was purchased from the National Collection of Type Cultures (Porton Down, UK). Ten strains of $S$. aureus were used: two community-acquired meticillin-resistant (MRSA) clinical isolates (CA3 and NRS384), one hospital-acquired MRSA clinical isolate (HA204), two vancomycin-intermediate resistant clinical isolates (5827 and 5836), the reference MRSA strain ATCC43300 (gifted by Dr. Peter Warn, University of Manchester, Manchester, UK), the "laboratory" MRSA strain BB270 and three "laboratory" meticillin-susceptible strains (Newman, SH1000 and RN4220). S. aureus strains were sourced as described in Desbois et al. [52].

Figure 2. Molecular structures of the six LC-PUFAs used in this present study. All double bonds are in cis orientation except for the $n-7$ bonds of 15-HETrE and 15-OHEPA that are in trans orientation.

DGLA

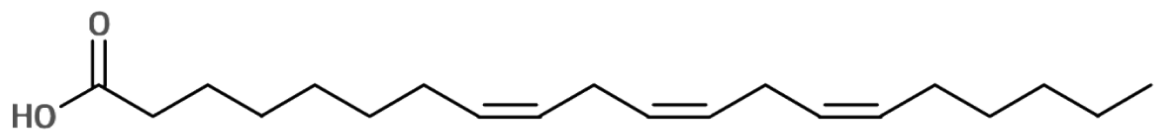

DHA

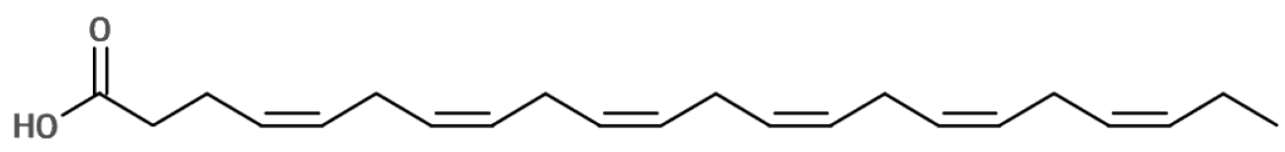

EPA

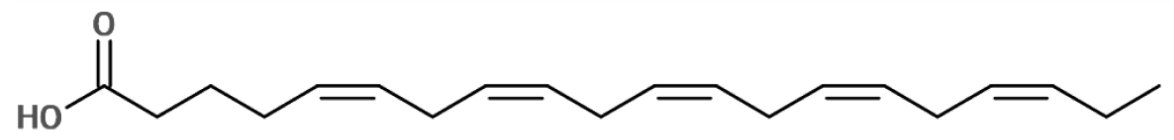

GLA

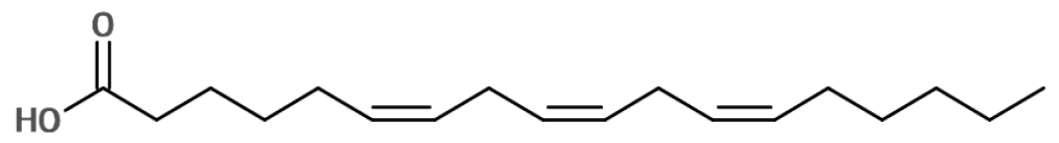

HETrE

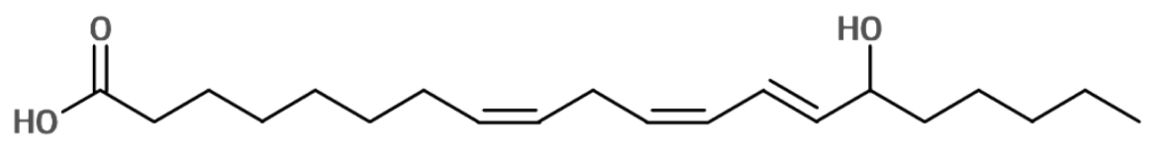

15-OHEPA

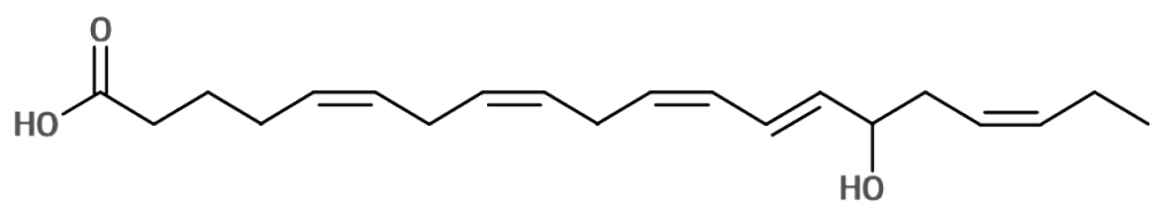




\subsection{MIC and MBC Determinations}

MICs and MBCs were determined for the six LC-PUFA and various clinical antimicrobial agents against $P$. acnes and $S$. aureus by broth micro-dilution according to protocols M07-A8 and M11-A5 published by the Clinical and Laboratory Standards Institute [53,54]. Doubling dilutions of the test compounds were prepared in sterile 96-well plates up to $4096 \mathrm{mg} / \mathrm{L}$. S. aureus experiments were performed in Mueller-Hinton broth (MHB), while $P$. acnes studies used supplemented brucella broth (SBB) [54]. Inoculums of $S$. aureus were prepared from late logarithmic phase cultures $(\sim 18 \mathrm{~h})$ that had been grown in $5 \mathrm{~mL}$ MHB at $37{ }^{\circ} \mathrm{C}$ with agitation at $140 \mathrm{rpm}$, while the $P$. acnes inoculum was prepared in 2-3 $\mathrm{mL}$ SBB by re-suspending colonies from supplemented brucella agar (SBA) plates that had been grown anaerobically $\left(10 \% \mathrm{CO}_{2}, 10 \% \mathrm{H}_{2}, 80 \% \mathrm{~N}_{2}\right)$ for $\sim 72 \mathrm{~h}$ at $37{ }^{\circ} \mathrm{C}$ (A85 Workstation; Don Whitley Scientific Ltd., Shipley, UK). Absorbance readings at $540 \mathrm{~nm}$ and $570 \mathrm{~nm}$ of the P. acnes and $S$. aureus suspensions were determined respectively, and bacterial inoculums were adjusted to $1 \times 10^{7} \mathrm{CFU} / \mathrm{mL}$ with fresh medium. Inoculum sizes were confirmed by diluting in phosphate-buffered saline (PBS) and plating on Mueller-Hinton agar (MHA) or SBA. Each well was inoculated with $5 \mu \mathrm{L}$ of bacterial suspension. Control wells contained media inoculated with bacterial suspension only, while negative control wells contained uninoculated culture medium only. Further inoculated wells controlled for the antimicrobial effects of the greatest concentrations of ethanol and DMSO used in the test wells $\left(4.5 \%\right.$ and $9.6 \%$, respectively). Microtitre plates were incubated at $37{ }^{\circ} \mathrm{C}$ for $18-20 \mathrm{~h}(48 \mathrm{~h}$ for $P$. acnes), and then the MICs were determined to be the lowest concentrations of each compound that prevented visible bacterial growth. MBCs were determined by plating $50 \mu \mathrm{L}$ from each well showing no visible growth on to MHA or SBA and incubating these plates at $37{ }^{\circ} \mathrm{C}$ for $48 \mathrm{~h}(96 \mathrm{~h}$ for P. acnes). The MBCs were the lowest concentrations of each compound that killed $\geq 99.9 \%$ of the initial inoculum. MIC and $\mathrm{MBC}$ determinations were performed in duplicate. $\mathrm{MIC}_{50}$ and $\mathrm{MBC}_{50}$ values were obtained by performing MICs and MBCs against the ten $S$. aureus isolates described above, and then determining the concentration required to inhibit or kill $50 \%$ of the strains, respectively.

\subsection{Kill Kinetics}

A S. aureus ATCC43300 inoculum was prepared as above, except that PBS was used to adjust the suspension to $1 \times 10^{7} \mathrm{CFU} / \mathrm{mL}$. The inoculum was diluted and plated on MHA to provide an accurate indication of CFU $/ \mathrm{mL}$. Solutions in PBS of the six LC-PUFAs were prepared at $1 \times \mathrm{MBC}$ and $300 \mu \mathrm{L}$ of each solution was dispensed to separate Eppendorf tubes. Controls contained just ethanol and DMSO diluted in PBS to the greatest concentration of each solvent used $(0.5 \%$ and $2.1 \%$, respectively). Each tube was inoculated with bacterial suspension $(15 \mu \mathrm{L})$ and incubated statically at $37^{\circ} \mathrm{C}$. At $15 \mathrm{~min}, 30 \mathrm{~min}, 1 \mathrm{~h}$ and $2 \mathrm{~h}, 10 \mu \mathrm{L}$ from each tube was plated on MHA in duplicate. Plates were incubated at $37{ }^{\circ} \mathrm{C}$ for $24 \mathrm{~h}$ and then $\mathrm{CFU}$ were quantified. The experiment was performed in triplicate.

\subsection{Checkerboard Tests}

Checkerboard assays were performed according to the standard protocol published by the American Society for Microbiology [55] to investigate the antimicrobial interactions between the six LC-PUFAs 
and two anti-acne agents (BPO and SA) against $P$. acnes. Further checkerboards were performed against $S$. aureus for BPO, SA, FUS, MUP, NEO and POL with these agents being assessed separately against each of the six LC-PUFAs. Doubling dilutions of each compound were prepared in MHB or SBB and dispensed to 96-well plates in the standard checkerboard pattern. Concentrations of each compound ranged from $\leq 1 / 16 \times$ MIC to $\geq 4 \times$ MIC. Control wells were dispensed with medium supplemented with the greatest concentration of solvent used in any well $(4.5 \%$ ethanol and/or $4.5 \% \mathrm{DMSO}$ ), while negative control wells contained just medium. Inoculums were prepared to $1 \times 10^{7} \mathrm{CFU} / \mathrm{mL}$ as above. Each well (except for negative controls) was inoculated with $5 \mu \mathrm{L}$ of bacterial suspension. Plates were incubated as for MIC determinations above and then the presence of bacterial growth was determined for each well according to the clarity of the medium and/or the presence of suspended colonies. The FIC for each well was determined for each interaction and the FICs from duplicate checkerboard assays were used to calculate a mean value that permitted interpretation of the nature of interaction between each combination of compounds.

\section{Conclusions}

There is a need for alternative agents to treat acne and superficial skin infections caused by $P$. acnes and $S$. aureus due to drug-resistance and undesirable side effects associated with certain clinical agents used at present. LC-PUFAs have attracted attention as new agents against these pathogens due to their potent antimicrobial actions and potential anti-inflammatory properties. This present study demonstrates that these natural compounds deserve further evaluation for the treatment of infections caused by $P$. acnes and $S$. aureus, and LC-PUFAs could be applied in combination with some currently available treatments to enhance therapeutic efficacy.

\section{Acknowledgments}

This work was funded by Dignity Sciences Ltd. who are pursuing the use of LC-PUFAs to treat acne. Dignity Sciences Ltd. had no influence over the study design; data collection, analysis and interpretation; and in the drafting of this article. This work was presented in part at the 44th Scottish Lipid Discussion Group Annual Meeting, University of Stirling, 14 June 2013. The authors would like to thank Wilf Mitchell (Heriot-Watt University, Edinburgh, UK) for access to laboratory equipment.

\section{Conflicts of Interest}

The authors declare no conflict of interest.

\section{References}

1. Bojar, R.A.; Holland, K.T. Acne and Propionibacterium acnes. Clin. Dermatol. 2004, 22, 375-379.

2. Harper, J.C. An update on the pathogenesis and management of acne vulgaris. J. Am. Acad. Dermatol. 2004, 51, S36-S38.

3. Motswaledi, M.H. Superficial skin infections and the use of topical and systemic antibiotics in general practice. S. Afr. Fam. Pract. 2011, 53, 139-142. 
4. Joint Formulary Committee. British National Formulary, 61st ed.; British Medical Association and Royal Pharmaceutical Society of Great Britain: London, UK, 2011.

5. Upton, A.; Lang, S.; Heffernan, H. Mupirocin and Staphylococcus aureus: A paradigm of emerging antibiotic resistance. J. Antimicrob. Chemother. 2003, 51, 613-617.

6. El-Zimaity, D.; Kearns, A.M.; Dawson, S.J.; Price, S.; Harrison, G.A.J. Survey, characterisation and susceptibility to fusidic acid of Staphylococcus aureus in the Carmarthen area. J. Antimicrob. Chemother. 2004, 54, 441-446.

7. Moon, S.H.; Roh, H.S.; Kim, Y.H.; Kim, J.E.; Ko, J.Y.; Ro, Y.S. Antibiotic resistance of microbial strains isolated from Korean acne patients. J. Dermatol. 2012, 39, 833-837.

8. Simonart, T.; Dramaix, M. Treatment of acne with topical antibiotics: Lessons from clinical studies. Br. J. Dermatol. 2005, 153, 395-403.

9. Desbois, A.P.; Smith, V.J. Antibacterial free fatty acids: activities, mechanisms of action and biotechnological potential. Appl. Microbiol. Biotechnol. 2010, 85, 1629-1642.

10. Desbois, A.P. Potential applications of antimicrobial fatty acids in medicine, agriculture and other industries. Recent Pat. Antiinfect. Drug Discov. 2012, 7, 111-122.

11. Berge, J.P.; Barnathan, G. Fatty acids from lipids of marine organisms: molecular biodiversity, roles as biomarkers, biologically active compounds, and economical aspects. Adv. Biochem. Eng. Biotechnol. 2005, 96, 49-125.

12. Desbois, A.P.; Mearns-Spragg, A.; Smith, V.J. A fatty acid from the diatom Phaeodactylum tricornutum is antibacterial against diverse bacteria including multi-resistant Staphylococcus aureus (MRSA). Mar. Biotechnol. 2009, 11, 45-52.

13. Desbois, A.P. Antimicrobial properties of eicosapentaenoic acid (C20: 5n-3). In Marine Microbiology: Bioactive Compounds and Biotechnological Applications; Kim, S.-K., Ed.; Wiley-VCH Verlag GmbH \& Co. KGaA: Weinheim, Germany, 2013; pp. 351-367.

14. Coonrod, J.D. Rôle of surfactant free fatty acids in antimicrobial defences. Eur. J. Respir. Dis. 1987, 153, 209-214.

15. Feldlaufer, M.F.; Knox, D.A.; Lusby, W.R.; Shimanuki, H. Antimicrobial activity of fatty acids against Bacillus larvae, the causative agent of American foulbrood disease. Apidologie 1993, 24, 95-99.

16. Maia, M.R.G.; Chaudhary, L.C.; Figueres, L.; Wallace, R.J. Metabolism of polyunsaturated fatty acids and their toxicity to the microflora of the rumen. Antonie Van Leeuwenhoek 2007, 91, 303-314.

17. Huang, C.B.; Ebersole, J.L. A novel bioactivity of omega-3 polyunsaturated fatty acids and their ester derivatives. Mol. Oral Microbiol. 2010, 25, 75-80.

18. Butcher, G.W.; King, G.; Dyke, K.G.H. Sensitivity of Staphylococcus aureus to unsaturated fatty acids. J. Gen. Microbiol. 1976, 94, 290-296.

19. Asthana, R.K.; Srivastava, A.; Kayastha, A.M.; Nath, G.; Singh, S.P. Antibacterial potential of $\gamma$-linolenic acid from Fischerella sp. colonising Neem tree bark. World J. Microbiol. Biotechnol. 2006, 22, 443-448.

20. Huang, C.B.; George, B.; Ebersole, J.L. Antimicrobial activity of $n-6, n-7$ and $n-9$ fatty acids and their esters for oral microorganisms. Arch. Oral Biol. 2010, 55, 555-560. 
21. Zhang, H.; Zhang, L.; Peng, L.; Dong, X.; Wu, D.; Wu, V.C.; Feng, F. Quantitative structure-activity relationships of antimicrobial fatty acids and derivatives against Staphylococcus aureus. J. Zhejiang Univ. Sci. B 2012, 13, 83-93.

22. Kristmundsdóttir, T.; Skúlason, S. Lipids as active ingredients in pharmaceuticals, cosmetics and health foods. In Lipids and Essential Oils as Antimicrobial Agents; Thormar, H., Ed.; John Wiley \& Sons, Ltd.: Philadelphia, PA, USA, 2011; pp. 151-177.

23. Mullen, A.; Loscher, C.E.; Roche, H.M. Anti-inflammatory effects of EPA and DHA are dependent upon time and dose-response elements associated with LPS stimulation in THP-1-derived macrophages. J. Nutr. Biochem. 2010, 21, 444-450.

24. Kalan, L.; Wright, G.D. Antibiotic adjuvants: multicomponent anti-infective strategies. Expert Rev. Mol. Med. 2011, 13, 1-17.

25. Odds, F.C. Synergy, antagonism, and what the chequerboard puts between them. J. Antimicrob. Chemother. 2003, 52, 1.

26. Ko, H.L.; Heczko, P.B.; Pulverer, G. Differential susceptibility of Propionibacterium acnes, P. granulosum and P. avidum to free fatty acids. J. Invest. Dermatol. 1978, 71, 363-365.

27. Nakatsuji, T.; Kao, M.C.; Fang, J.-Y.; Zouboulis, C.C.; Zhang, L.; Gallo, R.L.; Huang, C.M. Antimicrobial property of lauric acid against Propionibacterium acnes: its therapeutic potential for inflammatory acne vulgaris. J. Invest. Dermatol. 2009, 129, 2480-2488.

28. Decker, L.C.; Deuel, D.M.; Sedlock, D.M. Role of lipids in augmenting the antibacterialk activity of benzoyl peroxide against Propionibacterium acnes. Antimicrob. Agents Chemother. 1989, 33, 326-330.

29. Pannu, J.; McCarthy, A.; Martin, A.; Hamouda, T.; Ciotti, S.; Ma, L.; Sutcliffe, J.; Baker, J.R., Jr. In vitro antibacterial activity of NB-003 against Propionibacterium acnes. Antimicrob. Agents Chemother. 2011, 55, 4211-4217.

30. Blondeau, J.M. New concepts in antimicrobial susceptibility testing: the mutant prevention concentration and mutant selection window approach. Vet. Dermatol. 2009, 20, 383-396.

31. Shin, S.Y.; Bajpai, V.K.; Kim, H.R.; Kang, S.C. Antibacterial activity of bioconverted eicosapentaenoic (EPA) and docosahexaenoic acid (DHA) against foodborne pathogenic bacteria. Int. J. Food Microbiol. 2007, 113, 233-236.

32. Knapp, H.R.; Melly, M.A. Bactericidal effects of polyunsaturated fatty acids. J. Infect. Dis. 1986, 154, 84-94.

33. Nair, M.K.M.; Joy, J.; Vasudevan, P.; Hinckley, L.; Hoagland, T.A.; Venkitanarayanan, K.S. Antibacterial effect of caprylic acid and monocaprylin on major bacterial mastitis pathogens. J. Dairy Sci. 2005, 88, 3488-3495.

34. Bergsson, G.; Arnfinnsson, J.; Steingrímsson, Ó.; Thormar, H. Killing of Gram-positive cocci by fatty acids and monoglycerides. APMIS 2001, 109, 670-678.

35. Wille, J.J.; Kydonieus, A. Palmitoleic acid isomer (C16:1 $\Delta 6)$ in human skin sebum is effective against Gram-positive bacteria. Skin Pharmacol. Appl. Skin Physiol. 2003, 16, 176-187.

36. Clarke, S.R.; Mohamed, R.; Bian, L.; Routh, A.F.; Kokai-Kun, J.F.; Mond, J.J.; Tarkowski, A.; Foster, S.J. The Staphylococcus aureus surface protein isdA mediates resistance to innate defences of human skin. Cell Host Microb. 2007, 1, 1-14. 
37. Chamberlain, N.R.; Mehrtens, B.G.; Xiong, Z.; Kapral, F.A.; Boardman, J.L.; Rearick, J.I. Correlation of carotenoid production, decreased membrane fluidity, and resistance to oleic acid killing in Staphylococcus aureus 18Z. Infect. Immun. 1991, 59, 4332-4337.

38. Shin, S.Y.; Bajpai, V.K.; Kim, H.R.; Kang, S.C. Antibacterial activity of eicosapentaenoic acid (EPA) against foodborne and food spoilage microorganisms. LWT 2007, 40, 1515-1519.

39. Giamarellos-Bourboulis, E.J.; Grecka, P.; Dionyssiou-Asteriou, A.; Giamarellou, H. Impact of n-6 polyunsaturated fatty acids on growth of multidrug-resistant Pseudomonas aeruginosa: Interactions with amikacin and ceftazidime. Antimicrob. Agents Chemother. 2000, 44, 2187-2189.

40. Kitahara, T.; Aoyama, Y.; Hirakata, Y.; Kamihira, S.; Kohno, S.; Ichikawa, N.; Nakashima, M.; Sasaki, H.; Higuchi, S. In vitro activity of lauric acid or myristylamine in combination with six antimicrobial agents against methicillin-resistant Staphylococcus aureus (MRSA). Int. J. Antimicrob. Agents 2006, 27, 51-57.

41. Kravchenko, I.A.; Golovenko, N.Y.; Larionov, V.B.; Aleksandrova, A.I.; Ovcharenko, N.V. Effect of lauric acid on transdermal penetration of phenazepam in vivo. Bull. Exp. Biol. Med. 2003, 6, 579-581.

42. Saraiva, R.A.; Matias, E.F.F.; Coutinho, H.D.M.; Costa, J.G.M.; Souza, H.H.F.; Fernandes, C.N.; Rocha, J.B.T.; Menezes, I.R.A. Synergistic action between Caryocar coriaceum Wittm. fixed oil with aminoglycosides in vitro. Eur. J. Lipid Sci. Technol. 2011, 113, 967-972.

43. Chen, C.-H.; Wang, Y.; Nakatsuji, T.; Liu, Y.-T.; Zouboulis, C.C.; Gallo, R.L.; Zhang, L.; Hsieh, M.F.; Huang, C.M. An innate bactericidal oleic acid affective against skin infection of methicillin-resistant Staphylococcus aureus: A therapy concordant with evolutionary medicine. J. Microbiol. Biotechnol. 2011, 21, 391-399.

44. Huang, C.-M.; Chen, C.-H.; Pornpattananangkul, D.; Zhang, L.; Chan, M.; Hsieh, M.-F.; Zhang, L. Eradication of drug resistant Staphylococcus aureus by liposomal oleic acids. Biomaterials 2011, 32, 214-221.

45. Hart, R.; Bell-Syer, S.E.M.; Crawford, F.; Torgerson, D.J.; Young, P.; Russell, I. Systematic review of topical treatments for fungal infections of the skin and nails of the feet. BMJ 1999, 319, 79-82.

46. Georgel, P.; Crozat, K.; Lauth, X.; Makrantonaki, E.; Seltmann, H.; Sovath, S.; Hoebe, K.; Du, X.; Rutschmann, S.; Jiang, Z.; Bigby, T.; Nizet, V.; Zouboulis, C.C.; Beutler, B. A toll-like receptor 2-responsive lipid effector pathway protects mammals against skin infections with Gram-positive bacteria. Infect. Immun. 2005, 73, 4512-4521.

47. Drake, D.R.; Brogden, K.A.; Dawson, D.V.; Wertz, P.W. Antimicrobial lipids at the skin surface. J. Lipid Res. 2008, 49, 4-11.

48. Brogden, N.K.; Mehalick, L.; Fischer, C.L.; Wertz, P.W.; Brogden, K.A. The emerging role of peptides and lipids as antimicrobial epidermal barriers and modulators of local inflammation. Skin Pharmacol. Physiol. 2012, 25, 167-181.

49. Thormar, H.; Hilmarsson, H. The role of microbicidal lipids in host defense against pathogens and their potential as therapeutic agents. Chem. Phys. Lipids 2007, 150, 1-11. 
50. Fluhr, J.W.; Kao, J.; Jain, M.; Ahn, S.K.; Feingold, K.R.; Elias, P.M. Generation of free fatty acids from phospholipids regulates stratum corneum acidification and integrity. J. Invest. Dermatol. 2001, 117, 44-51.

51. Lee, D.-Y.; Huang, C.-M.; Nakatsuji, T.; Thiboutot, D.; Kang, S.-A.; Monestier, M.; Gallo, R.L. Histone $\mathrm{H} 4$ is a major component of the antimicrobial action of human sebocytes. J. Invest. Dermatol. 2009, 129, 2489-2496.

52. Desbois, A.P.; Gemmell, C.G.; Coote, P.J. In vivo efficacy of the antimicrobial peptide ranalexin in combination with the endopeptidase lysostaphin against wound and systemic meticillin-resistant Staphylococcus aureus (MRSA) infections. Int. J. Antimicrob. Agents 2010, 35, 559-565.

53. Clinical and Laboratory Standards Institute. Methods for Dilution Antimicrobial Susceptibility Tests for Bacteria That Grow Aerobically; Approved Standard M07-A8; Clinical and Laboratory Standards Institute: Wayne, PA, USA, 2008.

54. Clinical and Laboratory Standards Institute. Methods for Antimicrobial Susceptibility Testing of Anaerobic Bacteria; Approved Standard M11-A5; Clinical and Laboratory Standards Institute: Wayne, PA, USA, 2001.

55. American Society for Microbiology. Synergism testing: Broth microdilution checkerboard and broth macrodilution methods. In Clinical Microbiology Procedures Handbook; Isenberg, H.D., Ed.; ASM Press: Washington, DC, USA, 1992.

(C) 2013 by the authors; licensee MDPI, Basel, Switzerland. This article is an open access article distributed under the terms and conditions of the Creative Commons Attribution license (http://creativecommons.org/licenses/by/3.0/). 